\title{
Optimal Preparation of Drosophila Brain Samples for FIB-SEM
}

\author{
Lu, Z. ${ }^{1,2}$, Hayworth, K.J. ${ }^{1}$, Xu, S. ${ }^{1}$, Rivlin, P.K. ${ }^{1}$, \& Meinertzhagen, I.A. ${ }^{1,2}$ \\ 1. Flyem Team, Janelia Research Campus, HHMI, USA \\ 2. Department of Psychology and Neuroscience, Dalhousie University, Canada
}

Conventional chemical fixation followed by en bloc staining provides insufficient tissue contrast for FIB-SEM. To enhance membrane density, high-pressure freezing and freeze substitution (HPF-FS) and ferrocyanide reduced osmium-thiocarbohydrazide-osmium liganding (FRO-OTO) are commonly used, but each has its own shortcomings particularly for intact insect brain tissue with its dense packing of tiny axons. To map the neural pathways of the central nervous system (CNS) we developed a new procedure to prepare Drosophila brains with optimal contrast and morphological preservation for FIB-SEM. The method is abbreviated here as 'PLT-LS' and combines: Chemical fixation - a process incorporating progressive lowering of temperature [1] dehydration; low temperature en bloc staining; followed by room temperature infiltration and embedding. To give the tissue advanced intense staining and minimize artifacts, extraction, and size and shape variation, we treat tissue at temperature below $0{ }^{\circ} \mathrm{C}$ to $-25^{\circ} \mathrm{C}$ after routine fixation. We apply Karnovsky fixation for 2 hours then light osmium post-fixation, with $0.5 \% \mathrm{OsO}_{4}$ for $30 \mathrm{~min}$, and then dehydrate the tissue by gradually increasing the concentration of the dehydration agent from $0 \%$ to $97 \%$ and gradually decreasing the temperature from $0{ }^{\circ} \mathrm{C}$ to $-25^{\circ} \mathrm{C}$, and then incubating the tissue in acetone- or ethanol-based uranyl acetate and $\mathrm{OsO}_{4}$ at $-25^{\circ} \mathrm{C}$ for total around 40 hours. This helps to provide uniform osmication and staining, less proteins loss, less chance of distortion the fine structure, and more rigid tissue with less change in volume. The cutting property is reduced but tissue contrast is higher when using acetone compared with ethanol. Lead acetate, tannic acid, imidazole, phosphotungstic acid and organic solvent soluble dyes could be introduced into the low temperature en bloc staining protocol with fewer side-effects and more controllable staining. PLT-LS samples viewed in FIB-SEM images show highly visible membranes with fewer undulatory contours and conspicuous synaptic densities (Figure 1). For the best results we currently use FIB-SEM, PLT-LS samples with satisfactory hot-knife cutting properties [2] to derive a Drosophila brain connectome. This method works well on whole brains of adult Drosophila (Figure 2) and its first-instar larvae.

\section{References:}

[1] Patrick Echlin, Low-Temperature Microscopy and Analysis, Springer Science \& Business Media, New York 1992

[2] Kenneth J Hayworth et al, Nature Methods 12 (2015), p. 319. 

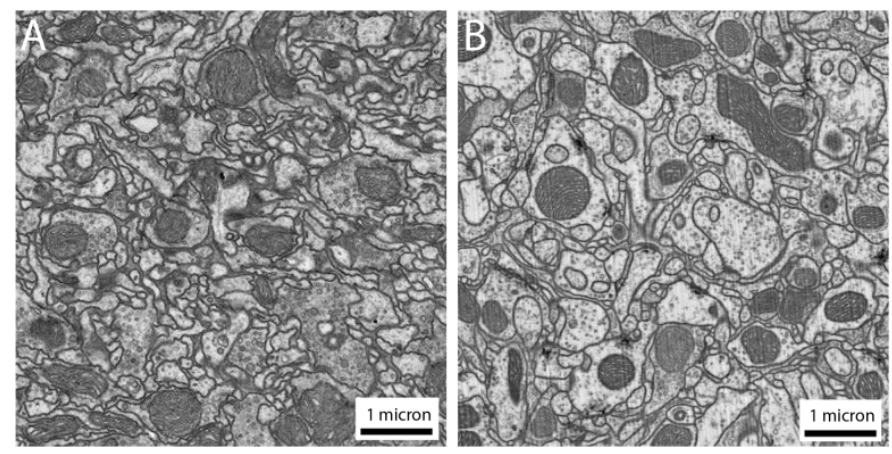

Figure 1. Comparison between Drosophila adult brain FIB-SEM samples of FRO-OTO and PLT-LS. A) Conventional fixation following by ferrocyanide and OTO membrane enhancement. B) Conventional fixation following by progressively lowered temperature dehydration and low temperature en bloc staining (PLT-LS). FIB-SEM data was acquired as 8x8x8nm voxels on a Zeiss Sigma.

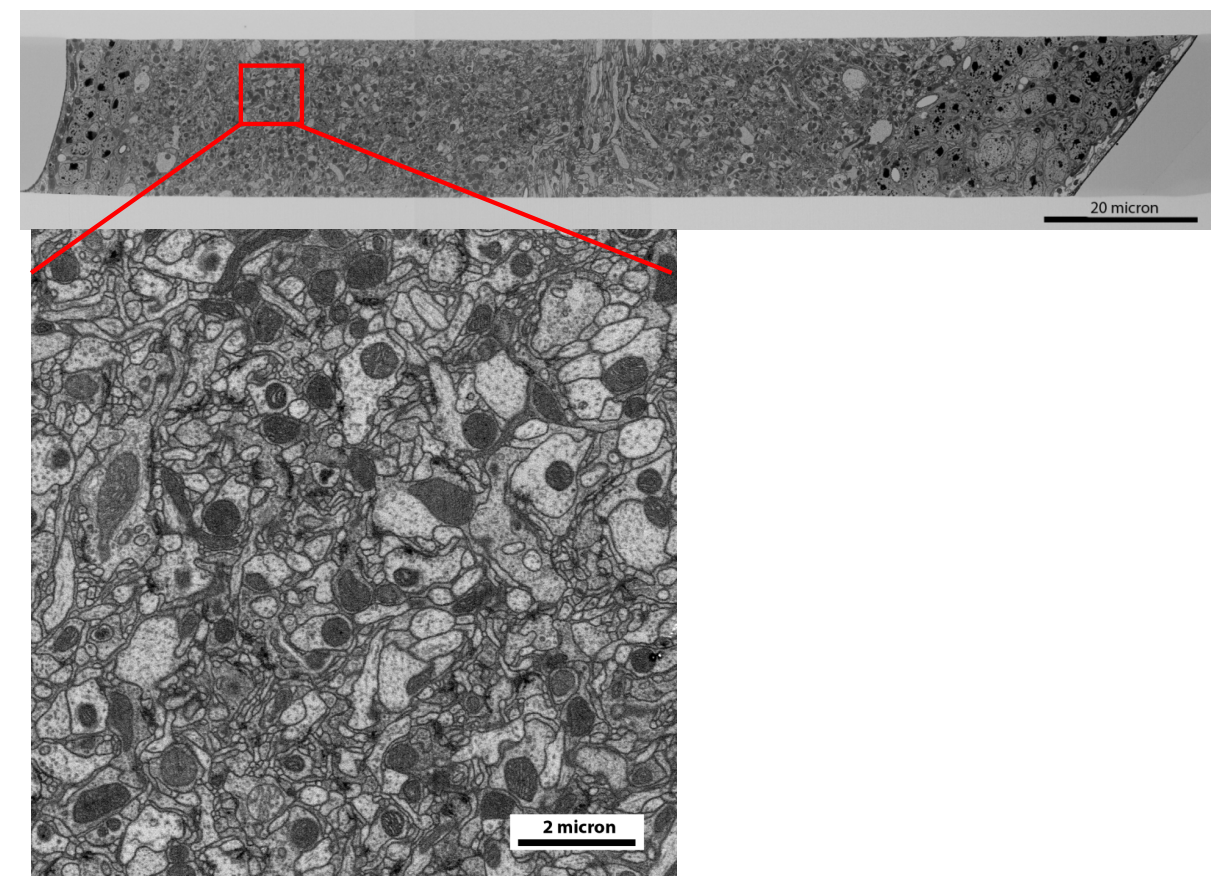

Figure 2. FIB-SEM image in cross section on $20 \mu \mathrm{m}$ thick hot-knife slices of a whole Drosophila adult brain. FIB-SEM data acquired at 8x8x8nm voxels using a Zeiss Sigma. 